\title{
Analysis of Pulmonary and Physical Function Three Months After Discharge for Moderate to Severe COVID-19
}

\author{
Bas Floris Maria Van Raaij ${ }^{1}$, Jordy Lauran Stöger ${ }^{2}$, Maarten Sebastiaan Werkman ${ }^{3}$, \\ Michiel Alexander De Graaf ${ }^{4}$, Maria Louisa Antoni ${ }^{4}$, Geert Hendrik Groeneveld ${ }^{5}$, \\ Anna Helena Elvire Roukens ${ }^{6}$, Frederikus Albertus Klok ${ }^{7}$, Soerindra Rajen Soeniel Ramai ${ }^{1}$, \\ Jacomina Jessica Miranda Geelhoed ${ }^{1, *}$ \\ ${ }^{1}$ Department of Pulmonary Diseases, Leiden University Medical Center, Leiden, The Netherlands \\ ${ }^{2}$ Department of Radiology, Leiden University Medical Center, Leiden, The Netherlands \\ ${ }^{3}$ Department of Physical Therapy, Leiden University Medical Center, Leiden, The Netherlands \\ ${ }^{4}$ Department of Cardiology, Leiden University Medical Center, Leiden, The Netherlands \\ ${ }^{5}$ Department of Acute Internal Medicine, Leiden University Medical Center, Leiden, The Netherlands \\ ${ }^{6}$ Department of Infectious Diseases, Leiden University Medical Center, Leiden, The Netherlands \\ ${ }^{7}$ Department of Medicine-Thrombosis and Hemostasis, Leiden University Medical Center, Leiden, The Netherlands
}

Email address:

j.j.m.geelhoed@lumc.nl (J. J. M. Geelhoed)

${ }^{*}$ Corresponding author

To cite this article:

Bas Floris Maria Van Raaij, Jordy Lauran Stöger, Maarten Sebastiaan Werkman, Michiel Alexander De Graaf, Maria Louisa Antoni, Geert Hendrik Groeneveld, Anna Helena Elvire Roukens, Frederikus Albertus Klok, Soerindra Rajen Soeniel Ramai, Jacomina Jessica Miranda Geelhoed. Analysis of Pulmonary and Physical Function Three Months After Discharge for Moderate to Severe COVID-19. Journal of Health and Environmental Research. Vol. 7, No. 3, 2021, pp. 145-153. doi: 10.11648/j.jher.20210703.15

Received: July 29, 2021; Accepted: August 11, 2021; Published: September 8, 2021

\begin{abstract}
Pulmonary fibrosis with persistent physiological deficit is a previously described feature of patients recovering from coronaviruses. Long-term health consequences of COVID-19 are still largely unknown. We aimed to evaluate whether CT abnormalities persist in COVID-19 survivors three months after discharge, and whether the amount of affected lung tissue correlates with pulmonary and physical function. Therefore, we evaluated patients three months after discharge at our outpatient clinic. All patients underwent pulmonary function testing, high resolution chest CT, six-minute walk test and handgrip strength test. We compared severely ill patients to moderately ill patients, whom respectively received treatment at the Intensive Care Unit and the general ward. In total 84 patients were included with a median age of $61 \cdot 4 \pm 12.9$ years of whom 53 were male. 50 patients had moderate disease and 34 were severely ill. 66 patients had residual abnormalities on follow-up chest CT. Reticulation and curvilinear bands were more frequent in severely ill patients (resp. $21 \%$ vs $4 \% ; p=0.029$ and $55 \%$ vs $23 \%$; $p=0 \cdot 004$ ). DLCOc, FEV1 and FVC in percentage of predicted were lower in severely ill patients. Severely ill patients were more likely to show an abnormal 6MWT, lower HGS and lower self-reported ADL and condition. In conclusion, in patients recovering from COVID-19, residual abnormalities were frequently present three months after hospitalization and associated with impaired pulmonary and physical function. This association was even stronger in patients who had been admitted to the ICU.
\end{abstract}

Keywords: COVID-19, Long-COVID, Multidisciplinary Follow-up, Outpatient Clinic, Pulmonary Function

\section{Introduction}

As of March 2021, the global pandemic of COVID-19, the infectious disease caused by Severe Acute Respiratory Syndrome Coronavirus 2 (SARS-CoV-2), has resulted in at least 120 million confirmed cases and more than 2.7 million deaths worldwide [1]. These numbers continue to increase daily. Recently, we published data on the short-term effects of acute COVID-19 up to six weeks after hospitalization [2]. Dyspnea on exertion was the most frequently reported 
symptom to persist. Furthermore, severely ill patients showed a lower pulmonary diffusion capacity. In another recent Dutch cohort study, residual pulmonary parenchymal abnormalities were present in more than $90 \%$ of discharged patients and correlated with lower diffusion capacity at three months follow-up [3]. In addition, a large Chinese study demonstrated that more severely ill patients were at higher risk of developing pulmonary diffusion disorders, fatigue or muscle weakness six months after onset [4]. These results are in line with previous observations in survivors of the severe acute respiratory syndrome coronavirus (SARS-CoV) and the Middle East respiratory syndrome coronavirus (MERS-CoV), of whom almost $30 \%$ showed impaired pulmonary function and abnormal radiologic findings consistent with fibrotic lung disease $[5,6]$.

A few additional studies focusing on Long-COVID found persisting symptoms and chest abnormalities in a significant proportion of patients $[7,8]$. Recently, the post-acute COVID-19 syndrome was described, which describes the persistent symptoms and/or complications beyond 4 weeks from the onset of symptoms [9]. Importantly, the long-term health consequences of COVID-19 are still largely unknown. Since dyspnea and fatigue were the most reported symptoms to persist, we aimed to evaluate the amount of CT abnormalities in COVID-19 survivors beyond 3 months after discharge, and whether the amount of affected lung tissue correlates with pulmonary and physical function.

\section{Methods}

\subsection{Design and Study Population}

This single-center prospective observational study was performed to evaluate long-term respiratory effects in patients recovering from acute COVID-19 after admission at the Leiden University Medical Center (LUMC), Leiden, the Netherlands. Patients were admitted between March $23^{\text {rd }}$ and June $23^{\text {rd }} 2020$ and were followed-up for 12 weeks after discharge. Patients older than 18 years living in the Leiden area were eligible after diagnosis of COVID-19 pneumonia was confirmed based on a positive polymerase chain reaction (PCR) test-result for SARS-CoV-2 in combination with the presence of typical radiological findings according to the COVID-19 Reporting and Data System (CO-RADS) [10]. Demographic and clinical characteristics were collected from medical reports according to the International Severe Acute Respiratory and emerging Infections Consortium (ISARIC) protocol [11]. Radiological reports of initial computed tomography (CT) were used to score all patients for the presence or absence of five distinctive findings: parenchymal consolidation, ground-glass opacities (GGO), reticulation, airway distortion and curvilinear bands. Furthermore, CORADS classification, total estimated percentage of affected lung parenchyma and CT Severity Score in total and per individual lobe were collected from CT reports when available. Patients were divided in two groups according to disease severity: i.e. severely ill and moderately ill patients.
Severe disease was defined as requiring ICU admittance, independent of respiratory therapy. Moderate disease involved all other patients who were treated on the general ward.

\subsection{Aims}

In this multidisciplinary cohort study we aimed to perform a systematic follow-up after hospitalization of COVID-19 patients and thereby identify the trajectory of physical symptom burden and recovery of imaging.

\subsection{Pulmonary Evaluation}

Patients were invited for follow-up visits 6 and 12 weeks after discharge. Pulmonary function tests (PFTs) were performed by technicians at the pulmonary function laboratory at both appointments. Forced expiratory volume in one second (FEV1), forced vital capacity (FVC) and diffusion capacity of the lungs for carbon monoxide adjusted for hemoglobin (DLCOc) were measured to assess obstructive and restrictive pulmonary diseases and diffusion disorders [12]. Follow-up imaging occurred after 12 weeks. Patients previously diagnosed with pulmonary embolism underwent pulmonary angiography with subtraction iodine maps; otherwise non-enhanced chest CT was acquired. All scans were prospectively evaluated by local radiologists as part of regular care. These radiological reports were used to score for the presence or absence of five distinctive parenchymal abnormalities as previously described for initial CT. To evaluate resorption rate of radiological abnormalities, all studies were semi-quantitative assessed by one radiologist in a manner analogous to the initial CT Severity Score on admission. Importantly, this measure reflects the area but not the degree (i.e. density) of affected parenchyma.

\subsection{Physical Function and Fitness}

Functional performance status was assessed with a sixminute walking test (6MWT), measuring six-minute walking distance (6MWD) as a measure for physical fitness, and handgrip strength (HGS) as a measure of overall skeletal muscle strength. 6MWT and HGS were performed following previously published protocols [13, 14]. Desaturation upon 6MWT was defined as a saturation of $<95 \%$. HGS was measured three times in the dominant hand with a handgrip dynamometer (JAMAR Handgrip Dynamometer; Sammons Preston Rolyan, Bolingbrook, IL). 6MWD and HGS were presented in meters and kilograms, respectively, and as percentage (\%) of predicted based on normative values [15]. A lower limit of $80 \%$ of predicted was used for both measures as cut-off value for classification of 'normal' or 'abnormal/decreased' [16].

Additionally, patients were asked to report ratings of their self-perceived physical fitness, ability to perform activities of daily living (ADL) and rate of recovery compared to preCOVID. Physical fitness and ADL were reported on a numeric ranging scale (NRS) ranging from 0 to 10 with higher scores reflecting higher levels of self-perceived fitness 
and function. Rate of recovery was scored using a Global Perceived Effect (GPE) rating. GPE expresses rate of recovery on a seven-point Likert scale ranging from 'complete recovery' to 'worse than ever' (Appendix).

\subsection{Statistical Analysis}

Demographic clinical characteristics and health effects of COVID-19 were analysed with descriptive statistics. Subgroup analysis based on ICU admission was performed with an independent T-test or Mann Whitney $U$ test for continuous variables. Categorical variables were tested with a Chi-Square test or Fischer's exact test. Pulmonary function tests of 6 and 12 weeks were compared with a paired T-test. The reported p-values are two-sided and are not corrected for multiple testing. Missing data was handled with completecase analysis. All statistical analyses were performed using IBM SPSS Statistics, version 25.

\subsection{Reporting and Ethics}

The study was approved by the hospital's ethical review board (Ethical Committee for COVID-19 related research at the LUMC, protocol number 2020-059). Research data were pseudonymized and securely stored, according to the General Data Protection Regulation. All patients admitted to the hospital were given a letter which stated that their data could be used for research purposes, and that they could opt out if they disagreed. None of the admitted patients have declined consent.

\subsection{Role of the Funding}

There was no external funding for this investigator driven analysis.

\section{Results}

A total of 175 patients with COVID-19 infection were admitted to the LUMC from March $23^{\text {rd }}$ to June $23^{\text {rd }}, 2020$, of which 140 patients could eventually be discharged. Of these patients, 56 were excluded because they were unable to attend follow-up appointments in the LUMC. Two patients were discharged to a hospice for palliative care. Thirty-six patients originated from another region and were scheduled for follow-up elsewhere. Seven patients declined follow-up testing, mainly due to absence of symptoms. Eight patients were lost to follow-up, because of administrative errors and three patients were re-admitted before the first outpatient consultation. The remaining 84 patients were included in the current analysis (Figure 1).

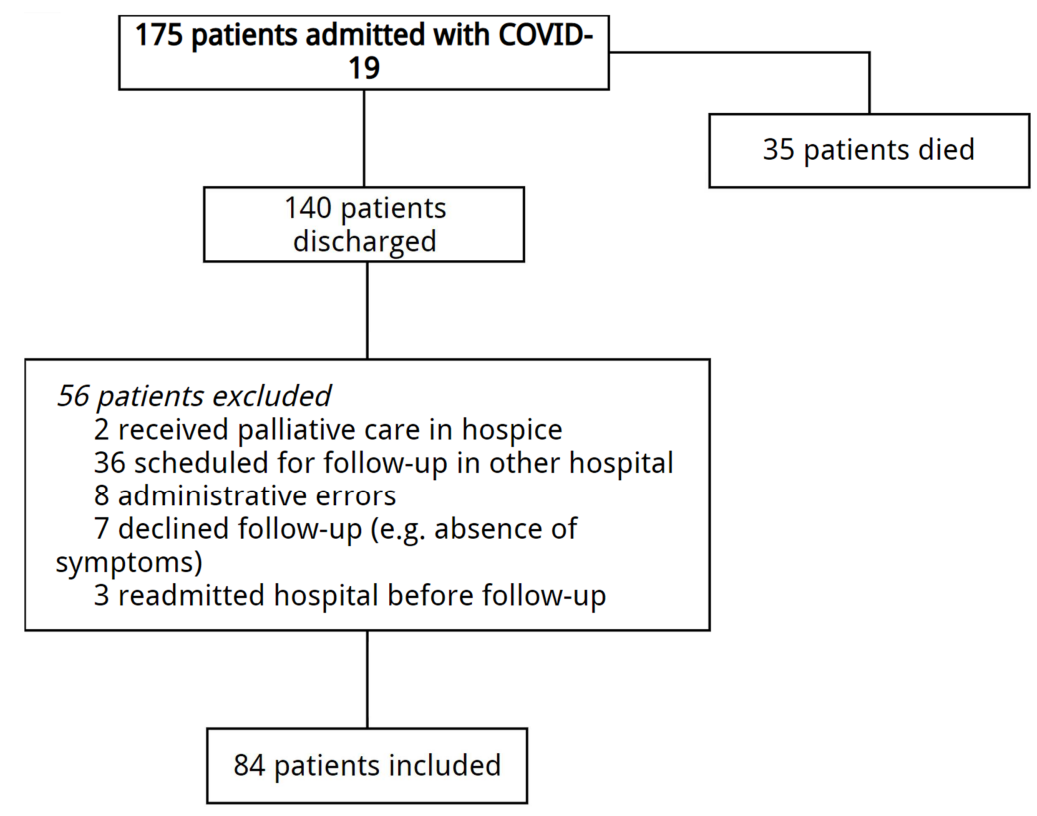

Figure 1. Enrollment of patients with COVID-19 and inclusion in the primary analysis.

Mean age $( \pm \mathrm{SD})$ of all patients was $61.4 \pm 12.9$ years with a range of 27-89 years, and $63 \%$ of patients were male (Table 1 ). A history of hypertension was present in $30 \%$ of patients, diabetes in $20 \%$, cardiovascular disease in $16 \%$, chronic lung disease in $17 \%$, with absence of pulmonary fibrosis. Estimated total affected lung tissue on initial chest $\mathrm{CT}$ was $33 \pm 21 \%$ with a median CT Severity Score of 11 (IQR: 9-15). GGO were present in $95 \%$ of patients, parenchymal consolidation in $83 \%$, reticulation in $21 \%$, curvilinear bands in $17 \%$ and airway distortion in $11 \%$ (Figure $2 \mathrm{~A}$ ). Thirty-four $(40 \%)$ patients were admitted to the ICU of whom 32 (97\%) required mechanical ventilation. Severely ill patients were younger compared to moderately ill patients $(57.6 \pm 11.1$ vs $63.9 \pm 13.5$ years; $\mathrm{p}=0.026)$. Notably, there was no difference in mean BMI (27.6 \pm 4.2 vs. $\left.27.2 \pm 5.1 \mathrm{~kg} / \mathrm{m}^{2} ; \mathrm{p}=0.692\right)$. In addition, sex, comorbidities, smoking status (i.e. never, former, active) and presence of distinctive radiologic abnormalities were comparable between subgroups. Severely ill patients had a higher median CT Severity Score (15, IQR: $11-21$ vs. 10 , IQR: $8-12$; $p=0.000$ ). Lobar involvement of COVID-19 on chest CT is shown in 
Figure 3A. Incidence of pulmonary embolisms was higher in

patients treated on the ICU ( $35 \%$ vs. $16 \% ; \mathrm{p}=0.042)$.
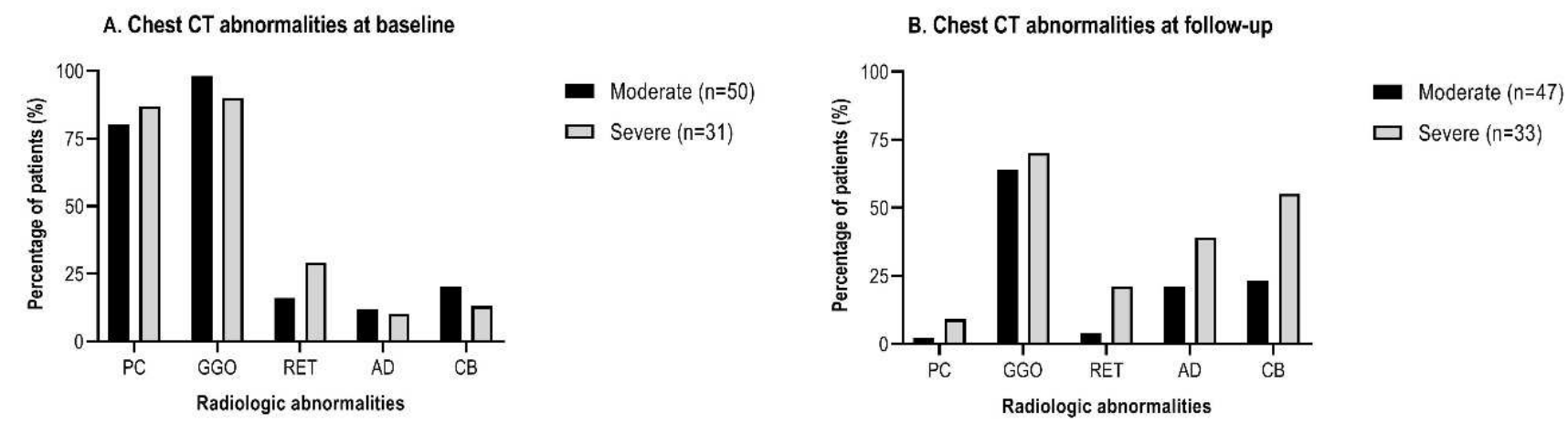

Figure 2. Presence of radiologic abnormalities on chest $C T$.

At baseline, prevalence of radiologic abnormalities is comparable between subgroups (panel A). Reticulation and curvilinear bands were more frequently seen in severely ill patients at follow-up when compared to moderately ill patients. $\mathrm{PC}=$ Parenchymal Consolidation. GGO=Ground-glass Opacities. $\mathrm{RET}=$ Reticulation. $\mathrm{AD}=$ Airway distortion. $\mathrm{CB}=$ Curvilinear Bands.

A. Lobar involvement on chest $\mathrm{CT}$ at baseline

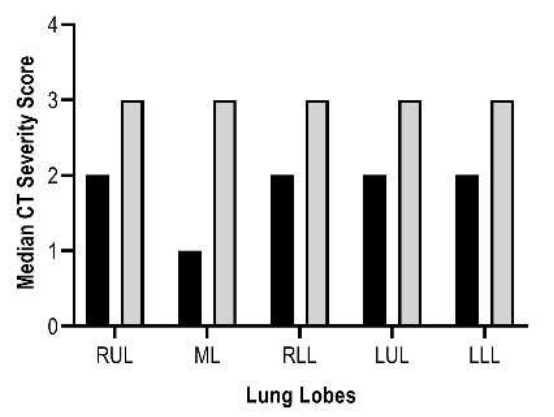

B. Lobar involvement on chest $\mathrm{CT}$ at follow-up

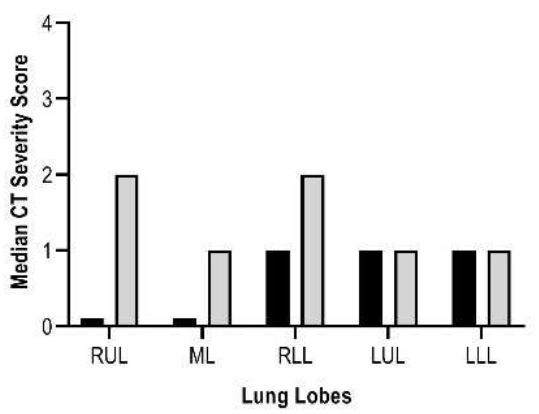

- Moderate $(\mathrm{n}=47)$

$\square$ Severe $(n=33)$

Figure 3. Lobular involvement of COVID-19 on chest CT.

All long lubes were involved at baseline independent of disease severity (panel A). Severely ill patients had a higher median CT Severity Score in all lobes. The median CT Severity Score declines in all patients an across all lobes at follow-up when compared to baseline (panel B). RUL=Right Upper Lobe. ML=Middle Lobe. RLL=Right Lower Lobe. LUL=Left Upper Lobe. $L L L=$ Left Lower Lobe.

\subsection{Follow-up Pulmonary Function and CT-imaging}

Pulmonary function tests and mean levels of CRP and ferritin 6 weeks after discharge are shown in Table 2. Mean DLCOc, FEV1 and FVC in\% of predicted were lower in severely ill patients. Overall, patients demonstrated significant improvement of mean DLCOc and mean FVC between 6 and 12 weeks after discharge (resp. $80.71 \pm 14.8 \%$ to $86.5 \pm 15.0 \%$ of predicted; $\mathrm{p}=0.001$ and from $92.7 \pm 19.2 \%$ to $95.9 \pm 19.7 \%$ of predicted; $\mathrm{p}=0.003$ ). There was no increase in FEV1. After 12 weeks, severely ill patients still demonstrated a lower FEV1 (mean difference of $13.8 \%$ of predicted; $p=0.001$ ), FVC (mean difference of $14.7 \%$ of predicted; $\mathrm{p}=0.001$ ) and DLCOc (mean difference of $14.8 \%$ of predicted; $p=0.000$ ) as shown in Table 3. Interestingly, moderately ill and severely ill patients showed similar DLCOc recovery rates between 6 and 12 weeks. Previous presence of pulmonary embolism was not associated with a lower DLCOc.

Follow-up chest $\mathrm{CT}$ at 12 weeks showed residual parenchymal abnormalities in $66(83 \%)$ patients. Overall median CT-severity score declined from 11 at baseline to
5 (IQR: 1-12). GGO were seen in $66 \%$ of patients, curvilinear bands in $36 \%$, airway distortion in $29 \%$, reticulation in $11 \%$ and parenchymal consolidations in $5 \%$. Residual abnormalities were more frequent in severely ill patients when compared to moderately ill patients $(94 \%$ vs. $75 \% ; \quad p=0.024)$. Prevalence of parenchymal consolidations, GGO and airway distortion were comparable between groups (Figure 2B). Reticulation and curvilinear bands were considerably more frequent in severely ill patients (resp. $21 \%$ vs. $4 \%$; $\mathrm{p}=0.029$ and $55 \%$ vs. $23 \%$; $=0.004)$. Median CT Severity Score declined from 15 to 10 (IQR: 4-13) in severely ill patients and from 10 to 3 (IQR: 0-7) in moderately ill patients. Figure $3 \mathrm{~B}$ shows lobar parenchymal involvement on follow-up chest CT.

Of all 20 patients who were diagnosed with pulmonary embolism, 8 were subjected to enhanced CT during followup; 7 showed completed resolution of the pulmonary emboli and 1 showed a persistent clot. None of the scans showed signs of chronic thromboembolic pulmonary hypertension (CTEPH), and pulmonary hypertension was ruled out with echocardiography in all 20 patients. 


\subsection{Functional Status}

Patients demonstrated normal physical fitness with a mean 6MWD of $461 \pm 132$ meters $(84 \pm 21 \%$ of predicted $)$ and handgrip strength of $36 \pm 12 \mathrm{~kg}(96 \pm 28 \%$ of predicted). Five patients were physically unable to perform the 6MWD, three of whom had been admitted to the ICU. Sixteen patients had a $6 \mathrm{MWD}$ of $<80 \%$ of predicted, with significantly more patients in the severe disease group (11 vs. 5; $\mathrm{p}=0.004)$. Desaturation during 6MWD was observed in 15 patients equally distributed between subgroups $(p=0.930)$. Severely ill patients reported significantly lower ADL and condition (resp. $6.7 \pm 1.6$ vs $7.8 \pm 1.9 ; \mathrm{p}=0.029,5.4 \pm 2.1$ vs. $6.8 \pm 1.5$; $\mathrm{p}=0.007)$. Global perceived effect was scored similar between subgroups $(\mathrm{p}=0.316)$.

Table 1. Baseline characteristics of all patients, according to disease severity.

\begin{tabular}{|c|c|c|c|c|}
\hline \multirow{2}{*}{ Characteristic } & \multirow{2}{*}{$\begin{array}{l}\text { Total } \\
n=84\end{array}$} & \multirow{2}{*}{$\begin{array}{l}\text { Moderate (non-ICU) } \\
n=50\end{array}$} & \multirow{2}{*}{$\begin{array}{l}\text { Severe (ICU) } \\
\mathrm{n}=34\end{array}$} & \multirow[t]{2}{*}{ p-value } \\
\hline & & & & \\
\hline Age, years & $61.4 \pm 12.9$ & $63.9 \pm 13.5$ & $57.6 \pm 11.1$ & 0.026 \\
\hline Sex, male & $53(63)$ & $32(64)$ & $21(62)$ & 0.835 \\
\hline BMI, $\mathrm{kg} / \mathrm{m}^{2}$ & $27.4 \pm 4.6,\{80\}$ & $27.2 \pm 4.2,\{46\}$ & $27.6 \pm 5.1$ & 0.692 \\
\hline \multicolumn{5}{|l|}{ Comorbidities } \\
\hline Diabetes mellitus & $17(20)$ & $7(14)$ & $10(30)$ & 0.084 \\
\hline Hypertension & $25(30)$ & $16(32)$ & $9(27)$ & 0.586 \\
\hline Asthma & $11(13)$ & $4(8)$ & $7(21)$ & 0.111 \\
\hline COPD & $3(4)$ & $3(6)$ & 0 & 0.296 \\
\hline Pulmonary embolism & $1(1)$ & 0 & $1(3)$ & 0.405 \\
\hline Cardiovascular disease & $13(16)$ & $8(16)$ & $5(15)$ & 0.872 \\
\hline Myocardial infarction & $3(4)$ & $1(2)$ & $2(6)$ & 0.563 \\
\hline PCI or $\mathrm{CABG}$ & $8(10)$ & $4(8)$ & $4(12)$ & 0.546 \\
\hline Heart valve anomaly & $5(6)$ & $3(6)$ & $2(6)$ & 1 \\
\hline Transient ischemic attack & $3(4)$ & $1(2)$ & $2(6)$ & 0.563 \\
\hline Chronic kidney disease & $5(6)$ & $3(6)$ & $2(6)$ & 1 \\
\hline Immunodeficiency & $6(7)$ & $5(10)$ & $1(3)$ & 0.394 \\
\hline Hypercholesterolemia & $6(7)$ & $4(8)$ & $2(6)$ & 1 \\
\hline Smoking status & & & & 0.655 \\
\hline Never & $43 / 73(59)$ & $25 / 44(57)$ & $18 / 29(62)$ & \\
\hline Active & 0 & 0 & 0 & \\
\hline Former & $30 / 73(41)$ & $19 / 44(43)$ & $11 / 29(38)$ & \\
\hline \multicolumn{5}{|l|}{ Clinical characteristics } \\
\hline SARS-CoV-2 PCR test-result, positive & $81(96)$ & $47(94)$ & $34(100)$ & 0.347 \\
\hline $\max . \mathrm{C}$-reactive protein, $\mathrm{mg} / \mathrm{L}$ & $189.4(86.9-307.4)$ & $111.9(67.0-188.0)$ & $326.6(256.6-400.0)$ & 0.000 \\
\hline max. D-dimer, ng/mL & $2543(1043-5574),\{58\}$ & $1126(869-1922),\{26\}$ & $4972(2670-7804),\{32\}$ & 0.000 \\
\hline $\max$. Ferritin, ug/L & $1069(559-2049),\{75\}$ & $822(382-1316),\{41\}$ & $1955(764-2711)$ & 0.001 \\
\hline Length of hospital stay, days & $16.6 \pm 15.2$ & $7.2 \pm 4.2$ & $30.5 \pm 14.8$ & 0.000 \\
\hline Length of ICU stay, days & NA & NA & $19.8 \pm 12.6$ & NA \\
\hline Home & $55(66)$ & $39(78)$ & $16(47)$ & 0.003 \\
\hline Rehabilitation clinic & $29(35)$ & $11(22)$ & $18(53)$ & \\
\hline \multicolumn{5}{|l|}{ Chest CT } \\
\hline Time from symptom onset to CT, days & $12.7 \pm 7.2,\{78\}$ & $11.2 \pm 5.2,\{49\}$ & $15.4 \pm 9.2,\{29\}$ & 0.028 \\
\hline CO-RADS & & & & 0.027 \\
\hline Category $<4$ & $1 / 79(1)$ & $1 / 49(2)$ & 0 & \\
\hline Category 4 & $5 / 79(6)$ & $3 / 49(6)$ & $2 / 30(7)$ & \\
\hline Category 5 & $51 / 79(65)$ & $37 / 49(76)$ & $14 / 30(47)$ & \\
\hline Category 6 & $22 / 79(28)$ & $8 / 49(16)$ & $14 / 30(47)$ & \\
\hline Affected lung parenchym, $\%$ & $33 \pm 21$ & $24 \pm 14$ & $50 \pm 21$ & 0.000 \\
\hline CT Severity Score, total & $11(9-15),\{81\}$ & $10(8-12)$ & $15(11-21),\{31\}$ & 0.000 \\
\hline CT Severity Score, RUL & $2(2-3),\{66\}$ & $2(1-2),\{44\}$ & $3(3-4),\{22\}$ & 0.000 \\
\hline CT Severity Score, ML & $2(1-3),\{66\}$ & $1(1-2),\{44\}$ & $3(2-4),\{22\}$ & 0.000 \\
\hline CT Severity Score, RLL & $3(2-3),\{66\}$ & $2(2-3),\{44\}$ & $3(2-4),\{22\}$ & 0.002 \\
\hline CT Severity Score, LUL & $2(2-3),\{66\}$ & $2(1-3),\{44\}$ & $3(2-4),\{22\}$ & 0.000 \\
\hline CT Severity Score, LLL & $3(2-3),\{66\}$ & $2(2-3),\{44\}$ & $3(2-5),\{22\}$ & 0.009 \\
\hline Parenchymal consolidation & $67 / 81(83)$ & $40(80)$ & $27 / 31(87)$ & 0.412 \\
\hline Ground-glass opacities & $77 / 81(95)$ & $49(98)$ & $28 / 31(90)$ & 0.121 \\
\hline Reticulation & $17 / 81(21)$ & $8(16)$ & 9/31 (29) & 0.162 \\
\hline Airway distortion & $9 / 81(11)$ & $6(12)$ & 3/31 (10) & 1 \\
\hline Curvilinear bands & $14 / 81(17)$ & $10(20)$ & $4 / 31(13)$ & 0.550 \\
\hline
\end{tabular}




\begin{tabular}{lllll}
\hline \multirow{2}{*}{ Characteristic } & Total & Moderate (non-ICU) & Severe (ICU) & p-value \\
\cline { 2 - 5 } & $\mathbf{n = 8 4}$ & $\mathbf{n = 5 0}$ & $\mathbf{n = 3 4}$ \\
\hline Treatment & & & & \\
Invasive mechanical ventilation & $33(39)$ & $\mathrm{NA}$ & $33(97)$ & $\mathrm{NA}$ \\
Duration of mechanical ventilation, days & $\mathrm{NA}$ & $\mathrm{NA}$ & $15.2 \pm 10.3$ & $\mathrm{NA}$ \\
Corticosteroids & $3(4)$ & 0 & $3(9)$ & $\mathrm{NA}$ \\
Duration of corticosteroids, days & $21.0 \pm 7.9$ & $\mathrm{NA}$ & $21.0 \pm 7.9$ & $\mathrm{NA}$ \\
Remdesivir & $23(27)$ & $20(40)$ & $3(9)$ & 0.002 \\
Duration of remdesivir, days & $6.1 \pm 2.2$ & $5.8 \pm 2.1$ & $9.0 \pm 1.4$ & 0.078 \\
(Hydroxy)-chloroquine & $47(56)$ & $17(34)$ & $30(88)$ & 0.000 \\
Duration of (hydroxy)-chloroquine, days & $4.6 \pm 1.1$ & $4.4 \pm 0.8$ & $4.7 \pm 1.2$ & 0.135 \\
\hline
\end{tabular}

Data are mean $\pm \mathrm{SD}$, median $(\mathrm{IQR})$ or $\mathrm{N}(\%)$. Missing data is indicated with $\mathrm{n} / \mathrm{N}$ or $\{\mathrm{N}\}$. ICU=Intensive Care Unit. BMI=Body Mass Index. COPD $=$ Chronic Obstructive Pulmonary Disease. PCI=Percutaneous Coronary Intervention. CABG=Coronary Artery Bypass Graft. PCR=Polymerase Chain Reaction. $\mathrm{CT}=$ Computed Tomography. RUL=Right Upper Lobe. ML=Middle Lobe. RLL=Right Lower Lobe. LUL=Left Upper Lobe. LLL=Left Lower Lobe. NA=Not Applicable.

Table 2. State of SARS-CoV-2 infection and pulmonary function at follow-up after 6 weeks.

\begin{tabular}{lllll}
\hline & Total & Moderate (non-ICU) & Severe (ICU) & p-value \\
\hline C-reactive protein, mg/L & $1.3(0.6-2.8),\{65\}$ & $1.1(0.6-2.2),\{39\}$ & $2(0.7-3.0),\{26\}$ & 0.254 \\
Ferritin, ug/L & $197(87-367),\{75\}$ & $180(96-362),\{44\}$ & $199(48-414),\{31\}$ & 0.821 \\
SARS-CoV-2 IgG antibodies, positive & $81(96),\{84\}$ & $48(96),\{50\}$ & $33(97),\{34\}$ & 0.797 \\
FEV1, L & $3.02 \pm 0.87,\{82\}$ & $3.15 \pm 0.85,\{49\}$ & $2.84 \pm 0.88,\{33\}$ & NA \\
FEV1, \% of predicted & $94.8 \pm 19.5,\{82\}$ & $100.8 \pm 17.6,\{49\}$ & $85.8 \pm 19.0,\{33\}$ & 0.000 \\
FVC, L & $3.82 \pm 1.13,\{82\}$ & $4.00 \pm 1.06,\{49\}$ & $3.55 \pm 1.20,\{33\}$ & NA \\
FVC, \% of predicted & $92.6 \pm 20.5,\{82\}$ & $98.8 \pm 17.9,\{49\}$ & $83.5 \pm 20.9,\{33\}$ & 0.001 \\
FEV1/FVC & $79.8 \pm 7.4,\{82\}$ & $79.0 \pm 8.1,\{49\}$ & $81.0 \pm 6.2,\{33\}$ & NA \\
DLCOc, \% of predicted & $78.9 \pm 15.7,\{76\}$ & $84.0 \pm 14.3,\{46\}$ & $71.1 \pm 14.7,\{30\}$ & 0.000 \\
\hline
\end{tabular}

Data are mean $\pm \mathrm{SD}$, median (IQR) or $\mathrm{N}(\%)$. Missing data is indicated with $\{\mathrm{N}\}$. IgG=Immunoglobulin $\mathrm{G}$. ICU=Intensive Care Unit. FVC=Forced vital capacity. FEV1=Forced expiratory volume in one second. DLCOc=Diffusion capacity of the lungs for carbon monoxide adjusted for hemoglobin.

Table 3. Pulmonary function, chest CT and physical fitness at follow-up after 12 weeks.

\begin{tabular}{|c|c|c|c|c|}
\hline & Total & Moderate (non-ICU) & Severe (ICU) & p-value \\
\hline FEV1, L & $3.06 \pm 0.82,\{73\}$ & $3.23 \pm 0.83,\{42\}$ & $2.83 \pm 0.77,\{31\}$ & NA \\
\hline FEV1, $\%$ of predicted & $95.5 \pm 18.7,\{73\}$ & $101.4 \pm 17.1,\{42\}$ & $87.6 \pm 18.1,\{31\}$ & 0.001 \\
\hline $\mathrm{FVC}, \mathrm{L}$ & $3.96 \pm 1.11,\{73\}$ & $4.21 \pm 1.00,\{42\}$ & $3.61 \pm 1.16,\{31\}$ & NA \\
\hline $\mathrm{FVC}, \%$ of predicted & $95.7 \pm 19.6,\{73\}$ & $102.3 \pm 15.2,\{42\}$ & $86.7 \pm 21.5,\{31\}$ & 0.001 \\
\hline FEV1/FVC & $78.0 \pm 7.6,\{73\}$ & $76.8 \pm 8.0,\{42\}$ & $79.6 \pm 6.9,\{31\}$ & NA \\
\hline DLCOc, $\%$ of predicted & $86.0 \pm 15.4,\{72\}$ & $92.2 \pm 13.5,\{42\}$ & $77.4 \pm 13.9,\{30\}$ & 0.000 \\
\hline Time between initial and follow-up CT, days & $114.3 \pm 24.4,\{80\}$ & $107.1 \pm 21.8,\{47\}$ & $125.6 \pm 24.4,\{33\}$ & 0.001 \\
\hline Residual abnormalities & $66(83),\{80\}$ & $35(75),\{47\}$ & $31(94),\{33\}$ & 0.024 \\
\hline CT Severity Score, total & $5(1-12),\{80\}$ & $3(0-7),\{47\}$ & $10(4-13),\{33\}$ & 0.002 \\
\hline CT Severity Score, RUL & $1(0-3),\{80\}$ & $0(0-1),\{47\}$ & $2(1-3),\{33\}$ & 0.004 \\
\hline CT Severity Score, ML & $1(0-2),\{80\}$ & $0(0-1),\{47\}$ & $1(0-3),\{33\}$ & 0.003 \\
\hline CT Severity Score, RLL & $1(0-3),\{80\}$ & $1(0-2),\{47\}$ & $2(1-3),\{33\}$ & 0.002 \\
\hline CT Severity Score, LUL & $1(0-2),\{80\}$ & $1(0-1),\{47\}$ & $1(1-2),\{33\}$ & 0.009 \\
\hline CT Severity Score, LLL & $1(0-2),\{80\}$ & $1(0-2),\{47\}$ & $1(1-3),\{33\}$ & 0.049 \\
\hline Parenchymal consolidation & $4(5),\{80\}$ & $1(2),\{47\}$ & $3(9),\{33\}$ & 0.301 \\
\hline Ground-glass opacities & $53(66),\{80\}$ & $30(64),\{47\}$ & $23(70),\{33\}$ & 0.585 \\
\hline Reticulation & $9(11),\{80\}$ & $2(4),\{47\}$ & $7(21),\{33\}$ & 0.029 \\
\hline Airway distortion & $23(29),\{80\}$ & $10(21),\{47\}$ & $13(39),\{33\}$ & 0.078 \\
\hline Curvilinear bands & $29(36),\{80\}$ & $11(23),\{47\}$ & $18(55),\{33\}$ & 0.004 \\
\hline 6MWT, m & $461 \pm 132,\{49\}$ & $488 \pm 125,\{29\}$ & $423 \pm 136,\{20\}$ & NA \\
\hline$\%$ of predicted & $84 \pm 21,\{47\}$ & $89 \pm 19,\{28\}$ & $77 \pm 23\{19\}$ & 0.060 \\
\hline no. of patients $<80 \%$ of predicted & $16 / 47(34),\{52\}$ & $5 / 28(18),\{30\}$ & $11 / 19(58),\{22\}$ & 0.004 \\
\hline no. of patients with desaturation & $15 / 44(34),\{52\}$ & $9 / 26(35),\{30\}$ & $6 / 18(33),\{22\}$ & 0.093 \\
\hline HGS, kg & $35.8 \pm 12.4,\{52\}$ & $40.4 \pm 11.3,\{30\}$ & $29.6 \pm 11.2,\{22\}$ & NA \\
\hline$\%$ of predicted & $95.5 \pm 27.5,\{51\}$ & $103.8 \pm 21.6,\{29\}$ & $84.6 \pm 31.0,\{22\}$ & 0.012 \\
\hline no. of patients $<80 \%$ of predicted & $10(20),\{52\}$ & $3(10),\{30\}$ & $7(32),\{22\}$ & 0.079 \\
\hline Activities of daily living, NRS & $7(6-9),\{52\}$ & $8(7-10),\{30\}$ & $7(6-8),\{22\}$ & 0.043 \\
\hline Condition, NRS & $6(5-8),\{52\}$ & $7(6-8),\{30\}$ & $6(4-7),\{22\}$ & 0.016 \\
\hline Global perceived effect, (1-7) & $5(4-6),\{52\}$ & $5(4-5),\{30\}$ & $5(5-6),\{22\}$ & 0.069 \\
\hline
\end{tabular}

Data are mean $\pm \mathrm{SD}$, median $(\mathrm{IQR})$ or $\mathrm{N}(\%)$. Missing data is indicated with $\mathrm{n} / \mathrm{N}$ or $\{\mathrm{N}\}$. \# Median reduction of total CT Severity Score in comparison with chest $\mathrm{CT}$ at baseline. $\mathrm{CT}=$ Computed Tomography. NA=Not Applicable. FVC=Forced vital capacity. FEV1=Forced expiratory volume in one second. $\mathrm{DLCOc}=$ Diffusion capacity of the lungs for carbon monoxide adjusted for hemoglobin. $\mathrm{RUL}=\mathrm{Right}$ upper lobe. $\mathrm{ML}=\mathrm{Middle}$ lobe. $\mathrm{RLL}=\mathrm{Right}$ lower lobe . LUL=Left upper lobe. $L L L=$ Left lower lobe. $6 \mathrm{MWT}=$ Six minute walk test. NRS=Numeric Rating Scale $(0-10)$. 


\section{Discussion}

In this observational cohort study, we reported the health consequences of adult COVID-19 survivors after hospital discharge. At this point, a majority of patients $(83 \%)$ reveals residual parenchymal abnormalities on chest CT. Compared to baseline imaging, the GGO and parenchymal consolidation typically associated with acute COVID-19 had clearly diminished. In some, parenchymal abnormalities evolved to residual signs of organization and architectural distortion, which could suggest pulmonary fibrosis. Severely ill patients were more likely to have residual abnormalities, showing an increased CT-severity score and diffusion abnormality with a higher prevalence of reticulation and curvilinear bands, which may suggest pulmonary fibrosis. Since severely ill patients had a longer time between baseline and follow-up CT due to a longer hospital stay, the severity of the residual parenchymal abnormalities in the ICU group could be even underestimated. These results are in line with previous studies on SARS survivors $[5,17]$.

Baseline chest CT had revealed parenchymal involvement of all pulmonary lobes. At three months follow-up, this was still the case for severely ill patients, whilst moderately ill patients showed residual abnormalities to be located predominantly in the lower lobes. This could simply be a result from differences in disease course severity or reflect effects of acute respiratory distress syndrome (ARDS) and mechanical ventilation as previously documented in ARDS survivors [18]. The latter assertion would be supported by recent data suggesting similarities between COVID-19related ARDS and non-COVID-19-related ARDS [19]. Additionally, most of the patients who required mechanical ventilation were partly ventilated in prone position, which could explain the residual abnormalities both ventral and dorsal in the lower lobes, assuming that follow-up CT scans in ARDS survivors showed a reticular pattern with a striking anterior distribution [20]. In the beginning of the COVID-19 pandemic, as well as in our cohort, only a few patients were treated with steroids when admitted to the hospital. Nowadays, dexamethasone is standard of care in hospitalized COVID-19 patients, which could have an effect on the residual abnormalities [21]. Notably, the resolution rate of the diagnosed pulmonary embolisms was comparable to that was seen in non-COVID-19 pulmonary embolism patients, although the number of patients is too low to allow for accurate conclusions on this matter [22, 23].

Pulmonary function testing showed a decreased diffusion capacity in the more severely ill patients, even when this was corrected for alveolar volume. This could be due in part to residual parenchymal abnormalities. As pulmonary embolisms and vascular changes are more frequently seen in COVID-19 patients who were admitted to the ICU [24], this could be another explanation for the difference in diffusion capacity. However, in our study the correlation between parenchymal abnormalities and diffusion capacity maintained after correction for pulmonary embolism.
Although three months after COVID-19 functional status parameters as 6-MWD and HGS were relatively preserved for the total group, we found that patients admitted to the ICU had an increased risk for lower functional status. Likewise, self-reported ratings on physical function and physical fitness were worse in the severely ill patients. This is in line with two other recent post-COVID-19 studies [3, 4], as well as reports concerning previous SARS survivors, in which $40 \%$ experienced chronic fatigue [25]. Furthermore, the relatively long length of stay in the hospital, immobilization on the ICU, duration of mechanical ventilation and use of neuromuscular blocking agents in those with critical disease are known risk factors for decreased skeletal muscle mass and physical capacity [26] which could have played an additional role to a possible primary COVID-19 related effect on physical function. Interestingly, global perceived recovery was comparable between ICU and non-ICU patients which corresponds with a similar recovery trend in pulmonary function (DLCOc) and decline in CT Severity.

The results of our study showed that the most severe COVID survivors had more significant residual abnormalities at follow-up CT scans. Chest CT scan during hospitalization could be useful to stratify patients in predicting the risk of residual abnormalities and thereby could guide therapeutic strategies. In clinical practice it is not feasible to perform a chest CT in al COVID-19 survivors 3 months after discharge. One could argue that complaints of persisting dyspnea on exertion, coughing, tiredness will guide performing additional analyses with HRCT and pulmonary and physical function tests. If the chest $\mathrm{CT}$ shows radiological inflammatory lung disease (an organizing pneumonia phenotype) in combination with persistent physiological and functional deficit, treatment with steroids was well tolerated and associated with rapid significant improvement [27]. Whether bronchoalveolar lavage or lung biopsy are needed to differentiate which patients will benefit from steroids is an area for future research. In addition, recent studies showed that patient with an underlying fibrotic interstitial lung disease (ILD) have an increased risk of death from COVID19, particularly elderly males and those with poor lung function and obesity $[28,29]$. Patients with ILD should be counseled for their increased risk, with an emphasis on public health measures to prevent infection. However, further studies evaluating the need for antifibrotic and immunomodulatory therapy in ILD are needed.

This study is strengthened by our multidisciplinary and systematic approach of assessing COVID-19 patients after hospitalization. Due to multiple reasons, only less than $50 \%$ of the patients originally admitted to the hospital eventually formed the study population, which may have hampered generalizability. CT Severity Score describes the area, not the level/degree of parenchymal involvement, it could indicate abnormalities that are not clinically relevant. Furthermore, functional status was assessed in only a subgroup of patients, mainly because those who could not be evaluated were in a 
rehabilitation setting elsewhere. Our study is limited by its single-center design and relatively small number of patients. Handling of our results that are derived from a tertiary care center mainly concern generalizability and should therefore be performed with some caution.

\section{Conclusion}

The current study showed that at an average of three months after hospitalization of COVID-19 patients residual abnormalities were frequently present and were associated with a lower pulmonary function and lower physical function. This association was strongest in severely ill patients. Imaging patterns consistent with organisation and architectural distortion were present in a minority of the patients. It is unsure if and to what degree these abnormalities will resolve and how many patients will develop some level of pulmonary fibrosis on long-term follow-up. Future follow-up imaging of the chest and physical fitness and function scores will probably help us better understanding this new entity and to find predictors of a worse long-term outcome of COVID-19 patients to target.

\section{Declaration of Interests}

The authors declare that they have no competing interests.

\section{Funding}

There was no external funding for this investigator driven analysis.

\section{Acknowledgements}

M. L. Antoni, J. J. M. Geelhoed, F. A. Klok and A. H. E. Roukens are part of the Dutch COVID and Thrombosis Coalition (DC\&TC).

\section{Appendix: Questions for Assessment of Self-reported Physical Fitness and Recovery (Translated from Dutch)}

$A D L$

Rate your ability to perform activities of daily living from 0 to 10 (higher numbers indicate better function).

$\begin{array}{lllllllllll}0 & 1 & 2 & 3 & 4 & 5 & 6 & 7 & 8 & 9 & 10\end{array}$

\section{Physical fitness}

Rate your condition from 0 to 10 (higher numbers indicate higher levels of physical fitness).

$\begin{array}{lllllllllll}0 & 1 & 2 & 3 & 4 & 5 & 6 & 7 & 8 & 9 & 10\end{array}$

Global perceived recovery compared to pre-COVID-19 Please circle the number that best indicates your rate of recovery compared to pre-COVID-19 status.
1. Complete recovery

2. Much improvement

3. Slightly improved

4. Comparable with pre-covid status

5. Slightly worsened

6. Much worsened

7. Worse than ever

\section{References}

[1] World Health Organization. WHO Coronavirus disease (COVID-19) Dashboard. https://covid19.who.int. Published on June 9th 2021.

[2] De Graaf, M. A., et al., Short-term outpatient follow-up of COVID-19 patients: A multidisciplinary approach. EClinicalMedicine, 2021: p. 100731.

[3] Van den Borst, B., et al., Comprehensive health assessment three months after recovery from acute COVID-19. Clin Infect Dis, 2020.

[4] Huang, C., et al., 6-month consequences of COVID-19 in patients discharged from hospital: a cohort study. Lancet, 2021. 397 (10270): p. 220-232.

[5] Hui, D. S., et al., Impact of severe acute respiratory syndrome (SARS) on pulmonary function, functional capacity and quality of life in a cohort of survivors. Thorax, 2005. 60 (5): p. 401-9.

[6] Das, K. M., et al., Follow-up chest radiographic findings in patients with MERS-CoV after recovery. Indian J Radiol Imaging, 2017. 27 (3): p. 342-349.

[7] Mandal, S., et al., 'Long-COVID': a cross-sectional study of persisting symptoms, biomarker and imaging abnormalities following hospitalisation for COVID-19. Thorax, 2020.

[8] Carfi, A., et al., Persistent Symptoms in Patients After Acute COVID-19. JAMA, 2020. 324 (6): p. 603-605.

[9] Nalbandian, A., et al., Post-acute COVID-19 syndrome. Nat Med, 2021. 27 (4): p. 601-615.

[10] Prokop, M., et al., CO-RADS: A Categorical CT Assessment Scheme for Patients Suspected of Having COVID-19Definition and Evaluation. Radiology, 2020. 296 (2): p. E97E104.

[11] Simpson, C. R., et al., The UK's pandemic influenza research portfolio: a model for future research on emerging infections. Lancet Infect Dis, 2019. 19 (8): p. e295-e300.

[12] Graham BL, Steenbruggen I, Miller MR et al. Standardization of Spirometry 2019 Update. An Official American Thoracic Society and European Respiratory Society Technical Statement. Am J Respir Crit Care Med. 2019; 200 (8): e70e88.

[13] Holland, A. E., et al., An official European Respiratory Society/American Thoracic Society technical standard: field walking tests in chronic respiratory disease. Eur Respir J, 2014. 44 (6): p. 1428-46.

[14] Ali, N. A., et al., Acquired weakness, handgrip strength, and mortality in critically ill patients. Am J Respir Crit Care Med, 2008. 178 (3): p. 261-8. 
[15] Enright, P. L. and D. L. Sherrill, Reference equations for the six-minute walk in healthy adults. Am J Respir Crit Care Med, 1998. 158 (5 Pt 1): p. 1384-7.

[16] Dodds, R. M., et al., Grip strength across the life course: normative data from twelve British studies. PLoS One, 2014. 9 (12): p. e113637.

[17] Balbi, M., et al., Post-discharge chest CT findings and pulmonary function tests in severe COVID-19 patients. Eur J Radiol, 2021. 138: p. 109676.

[18] Herridge, M. S., et al., One-year outcomes in survivors of the acute respiratory distress syndrome. N Engl J Med, 2003. 348 (8): p. 683-93.

[19] Grasselli, G., et al., Pathophysiology of COVID-19-associated acute respiratory distress syndrome: a multicentre prospective observational study. Lancet Respir Med, 2020. 8 (12): p. 1201-1208.

[20] Desai, S. R., et al., Acute respiratory distress syndrome: CT abnormalities at long-term follow-up. Radiology, 1999. 210 (1): p. 29-35.

[21] Group, R. C., et al., Dexamethasone in Hospitalized Patients with COVID-19. N Engl J Med, 2021. 384 (8): p. 693-704.

[22] Klok, F. A., et al., Diagnosis of chronic thromboembolic pulmonary hypertension after acute pulmonary embolism. Eur Respir J, 2020. 55 (6).
[23] Huisman, M. V., et al., Pulmonary embolism. Nat Rev Dis Primers, 2018. 4: p. 18028.

[24] Dutch, C., et al., Incidence of thrombotic complications and overall survival in hospitalized patients with COVID-19 in the second and first wave. Thromb Res, 2021. 199: p. 143-148.

[25] Lam, M. H., et al., Mental morbidities and chronic fatigue in severe acute respiratory syndrome survivors: long-term follow-up. Arch Intern Med, 2009. 169 (22): p. 2142-7.

[26] Kress, J. P. and J. B. Hall, ICU-acquired weakness and recovery from critical illness. N Engl J Med, 2014. 371 (3): p. 287-8.

[27] Myall, K. J., et al., Persistent Post-COVID-19 Inflammatory Interstitial Lung Disease: An Observational Study of Corticosteroid Treatment. Ann Am Thorac Soc, 2021.

[28] Drake, T. M., et al., Outcome of Hospitalization for COVID19 in Patients with Interstitial Lung Disease. An International Multicenter Study. Am J Respir Crit Care Med, 2020. 202 (12): p. 1656-1665.

[29] Esposito, A. J., et al., Increased Odds of Death for Patients with Interstitial Lung Disease and COVID-19: A Case-Control Study. Am J Respir Crit Care Med, 2020. 202 (12): p. 17101713. 